\title{
Cytological, histochemical, and ultrastructural study of human foetal liver of various gestation with future implications in segmental resection: an anatomical perspective
}

\author{
Arpan Haldar ${ }^{1}$, Manisha Rajanand Gaikwad ${ }^{2}$, Apurba Patra $^{3}$, Soumya C. Bhattacharya ${ }^{4}$ \\ ${ }^{1}$ Department of Anatomy, All India Institute of Medical Sciences, Deoghar, ${ }^{2}$ Department of Anatomy, All India Institute of Medical Sciences, \\ Bhubaneswar, ${ }^{3}$ Department of Anatomy, All India Institute of Medical Sciences, Bathinda, ${ }^{4}$ Department of Anatomy, ESI-PGIMSR, Kolkata, India
}

\begin{abstract}
The liver is the largest gland of the gastrointestinal tract having both exocrine and endocrine functions. Developmentally it arises as a ventral outgrowth from the gut endoderm during 3rd week of intrauterine life. The foetal liver is very important because of its synthetic and hemopoietic potential. The present work aimed to see the detailed histogenesis and development of the foetal liver by cytological, immunohistochemical and ultrastructural study. The liver tissue of nine aborted foetuses of various gestational age were studied. For cytology: special stains like Masson trichrome, periodic acid Schiff and reticulin were used, immunohistochemical staining was performed with triple antibodies (c-myc, Ki-67 and Ber-H2), and for ultrastructure: aluminium mounted specimens were coated with gold and argon gas and observed under scanning electron microscopy (EM). Cytology and immunohistochemistry showed the development of duct patterns and hemopoietic patterns in all stages of fetogenesis. The ductal plate was marked by the layer of dark brown staining cells at the edge of two portal tracts. The haemopoietic cells with sinusoids and aggregation of hepatocytes were observed in the early weeks of gestation. EM showed tree-like branching of a portal canal depicting hepatic segmentation of foetal liver. The organizational changes in lobular pattern, duct pattern, and microstructure of liver during fetogenesis are very crucial to achieve the adult morphology in feature. Histogenesis of the foetal liver follows a multistep process depending upon the gestational age, any deviation from normalcy may lead to structural and functional abnormality later in life.
\end{abstract}

Key words: Hepatocytes, Immunohistochemistry, Cytology, Inclusion bodies

Received July 22, 2021; 1st Revised August 27, 2021; 2nd Revised August 28, 2021; 3rd Revised September 2, 2021; 4th Revised September 13, 2021; Accepted September 24, 2021

\footnotetext{
Corresponding author:

Apurba Patra (iD

Department of Anatomy, All India Institute of Medical Sciences, Bathinda 151001 , India

E-mail:apurba.cnmco3@gmail.com
}

\section{Introduction}

The liver is the largest gland of the human body consists of both exocrine and endocrine parts. The exocrine part of the liver secretes bile juice while the endocrine part secretes hormones responsible for glucose metabolism and also secretes most of the plasma proteins [1]. Embryologically, it arises as a ventral outgrowth from the gut endoderm during 3 rd week of intrauterine life [2]. It plays a pivotal role in the maintenance of body homeostasis by regulation of various 
physiological processes such as metabolism of carbohydrates and fats, bile acids synthesis, production of proteins, glycogen storage, etc [3]. The adult liver tends to have huge regenerative capacity and it is the best example of an internal human organ capable of natural regeneration of lost tissue [4]. In foetal life, the liver starts functioning which includes glycogen storage, decomposition of red blood cells, plasma protein synthesis, detoxification and haematopoiesis. To carry out these vital functions, the liver parenchyma must therefore be arranged in such a way so that every hepatocyte on one or more of its surfaces abuts on a passageway that connects with a duct system to carry away its exocrine secretion (bile) and abuts on a blood vessel into which it delivers its endocrine secretions [5]. These minute details about the cellular organization of liver parenchyma are really crucial during segmental resection of the liver in adult age due to hepatocellular carcinoma or cirrhosis. Various researchers [6, 7] have studied the histogenesis and morphogenesis of liver parenchyma but none of them has described the ultrastructure of the duct pattern concerning the gestational age. Also, no data is showing the immunoelectron microscopy of the human foetal liver which can be in future studies correlated with antibodies present in the liver. Although mesenchymal umbilical tissue has been in trials in hepatic tissue engineering for the treatment of end-stage liver disease to date there is no foetal liver transplantation in patients with cirrhosis and hepatocellular carcinoma due to the deficient knowledge of the descriptive histological architecture of the human foetal liver. With such a background, the present study (cytological, histochemical, and ultrastructural) of human foetal liver of various gestational ages was undertaken.

\section{Materials and Methods}

The liver tissue of nine aborted human foetuses of 1st, 2nd and 3rd trimesters of gestation which did not show any evidence of external morphological abnormality were collected from the Department of Gynaecology and Obstetrics, All India Institute of Medical Sciences, Bhubaneswar after therapeutic abortion, which was used in this study after approval of the Institutional Ethical Committee of Human Research (IEC) and Institute Research Board (IRB) of AIIMS, Bhubaneswar (Ref Number: T/IM-NF/Anatomy/18/84). They were collected after written informed consent of the legal guardian accorded with institutional guidelines, which is maintained in the foetal death register in the Department of Anatomy, AIIMS, Bhubaneswar. These foetuses included spontaneous abortions, stillborn foetuses, preterm babies and also a few autopsy specimens. Foetuses with a history of twin pregnancy or pregnancy-associated with metabolic disorders (diabetes mellitus, hypertension, and thyroid disorders), gross congenital anomalies or other birth defects were excluded from the study.

\section{For cytological study}

The foetuses were dissected by dissecting microscope and the liver tissue of 2-mm size were immediately fixed in $10 \%$ formalin for 24 hours. After fixation by formalin, the tissues were processed for routine histological procedures using special stains like Masson trichrome, periodic acid Schiff and reticulin stain and then photomicrographs will be taken by a camera using a microscope adapter.

\section{For immunohistochemical study}

Sections of liver tissues ( $3 \mathrm{~mm}$ ) tissues were fixed in 10\% neutral buffered formalin for 7 hours and then subjected to routine processing and paraffin embedding. Antigen retrieval from those tissues was performed by heating unstained sections which were immersed in DAKO Target Retrieval Solution (Dako North America Inc, Carpinteria, CA, USA). Deparaffinized sections were incubated with $3 \%$ hydrogen peroxide for 5 minutes which blocks and inhibit the endogenous enzymes to avoid producing non-specific binding of Antigen in the cell surface, followed by sequential 10-minute incubations of xylene and alcohol. It was put in the pressure cooker for 20 minutes (Heat-Induced Epitope Retrieval) to unmask the antigen epitopes to allow the antibodies to bind which was followed by a biotinylated link antibody and peroxidise-labelled streptavidin, which augmented the Antigen expression on the tissue surface. A modified labelled avidinbiotin immunohistochemical staining was performed with triple antibodies C-myc, Ki-67 and Ber-H2 were performed with Immunoperoxidase Kit (DAKO) on DAKO Auto Stainer. Staining was completed after a 10 -minute incubation with DAKO 3,3'-diaminobenzidine (DAB Chromogen Kit; Biocare Medical, Pacheco, CA, USA) chromogen, which was used as a signal enhancer. All cases were coded, and the grading of the immunostaining was performed on a sliding scale of $1+$ to $4+$ according to the percentage of reactive cells (0, no staining; $1+, 1 \%$ to $25 \% ; 2+, 26 \%$ to $50 \% ; 3+, 51$ to $75 \%$; $4+, \geq 76 \%)$. 


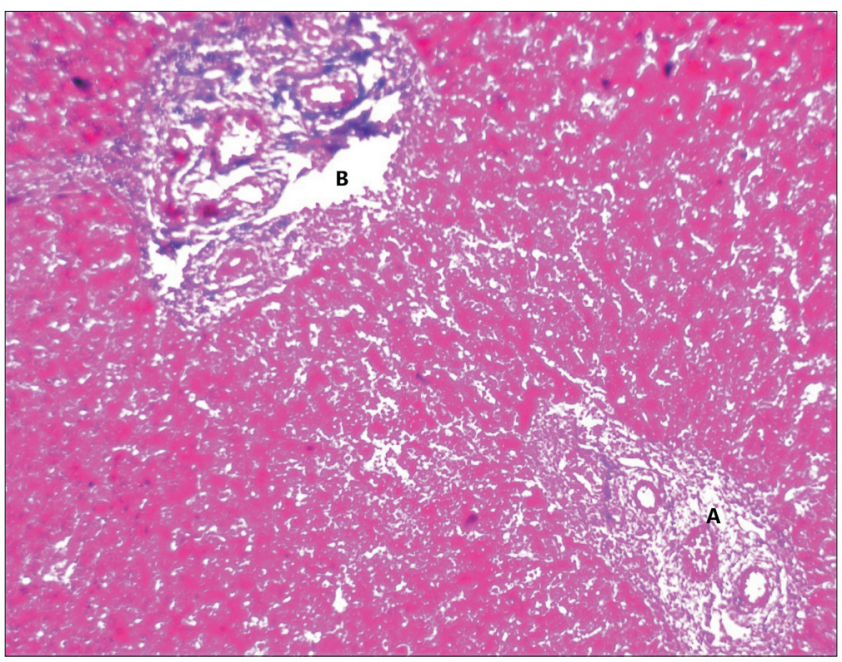

Fig. 1. Photomicrograph (Masson trichrome, $\times 10$ ) of cytology showing the formation of the portal triad (A) and future central vein (B) in 1st-trimester foetal liver.

\section{For ultrastructural study}

The liver tissue was cut in 3-mm size and was washed with three changes of $0.1 \mathrm{M}$ phosphate buffer 15 minutes each at $4^{\circ} \mathrm{C}$ and then the section was flooded with $1 \%$ osmium tetroxide to post-fix for 2 hours at $4^{\circ} \mathrm{C}$. Then it was dehydrated with three changes of acetone 15 minutes each and then 2 changes in dry acetone 15 minutes each and was transferred to liquid $\mathrm{CO}_{2}$ in a chamber that is cooled and put under pressure. When acetone has been completely removed and the tissues were impregnated with liquid $\mathrm{CO}_{2}$, the chamber was warmed up to critical point $31.5^{\circ} \mathrm{C}$ where the density of the drying medium is the same in both liquid and gas phases at 7,584 $\mathrm{kPa}$ (critical point drying).

After drying the specimens was then be mounted on aluminium stubs with conductive paint (silver or copper paint) with the area of interest exposed towards the surface and then coating was done with gold and argon gas was flooded in the chamber for 1 minute, ultimately a uniform thin layer was deposited on the specimen (sputter coating). After metal coating, the specimen was ready for observation under scanning electron microscope.

\section{Results}

The prenatal specimens were categorized into three trimesters, as per gestational age groups: week 1 to the end of 12 weeks (1st trimester), week 13 to the end of week 26 (2nd trimester), week 27 to the end of pregnancy (3rd trimester).

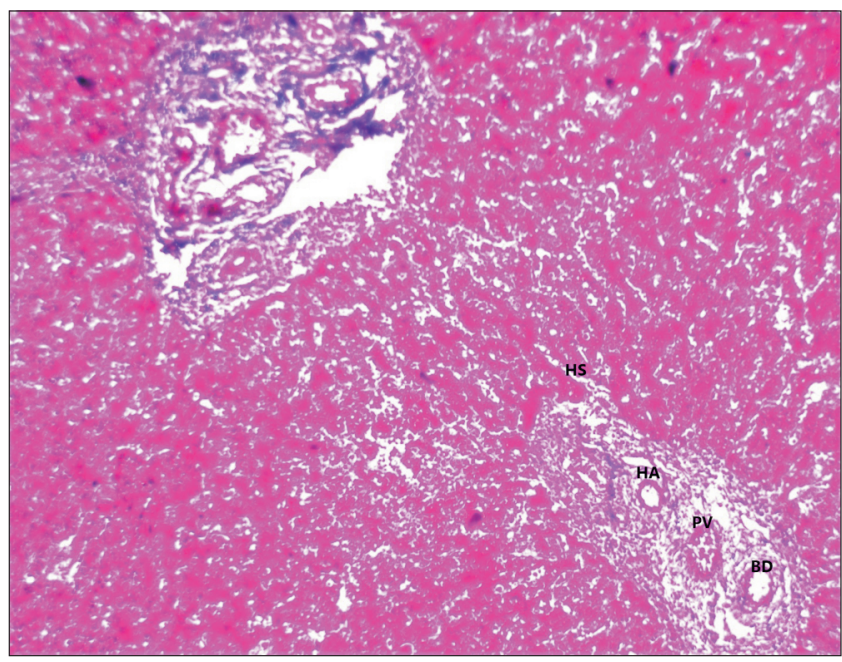

Fig. 2. Photomicrograph (Masson trichrome, $\times 10$ ) of cytology showing the formation of the portal triad (the hepatic artery [HA], portal vein $[\mathrm{PV}]$ and bile duct $[\mathrm{BD}]$, hepatic sinusoids [HS] in 2ndtrimester foetal liver.

\section{Cytological study}

\section{1st trimester}

In the early weeks' sinusoids and aggregation of hepatocytes around the central vein was seen. The central vein was well-formed with radiating cords of cells towards the periphery, the portal triad was just visible. Foetuses towards the end of this trimester showed hepatic lobule formation, scattered Kupffer cells on the margin of sinusoids and formation stage of radiating cords of hepatocyte (Fig. 1).

\section{2nd trimester}

Well-marked central vein with radiating cords of hepatocytes with reticular fibres and Kupffer cell were seen (Fig. 2).

\section{3rd trimester}

Increased number of reticular fibres with Kupffer cells, Central vein, radiating cords of hepatocytes, portal triad, Glisson's capsule or hepato-biliary capsule covering portal triad (Fig. 3).

\section{Immunohistochemical study}

The normal ductal plate, marked by the layer of dark brown staining cells at the edge of portal tracts (Fig. 4) was seen. It was multilaminar in places and contained a few lumina inside. Brownish colouration was due to immunopositivity to CD 30 (Ber-H2), c-myc, and Ki-67. Developing stromal 
cells, central vein and sinusoids showed immunopositivity to the labelling of CD 30, c-myc, and Ki-67. Immune expression towards c-myc was more prominent than Ber-H2 (Fig. 5) and of similar intensity in Ki-67 labelling (Fig. 6). The connective tissue and other structures of the portal tract were not specifically stained. Hepatocytes with medium density brown staining are evident between the two portal tracts and the central veins. Erythrocytes are seen in the hepatic sinusoids. Discontinuous, mostly unilaminar ductal plates are found around four portal areas of varying sizes. The bile ductule

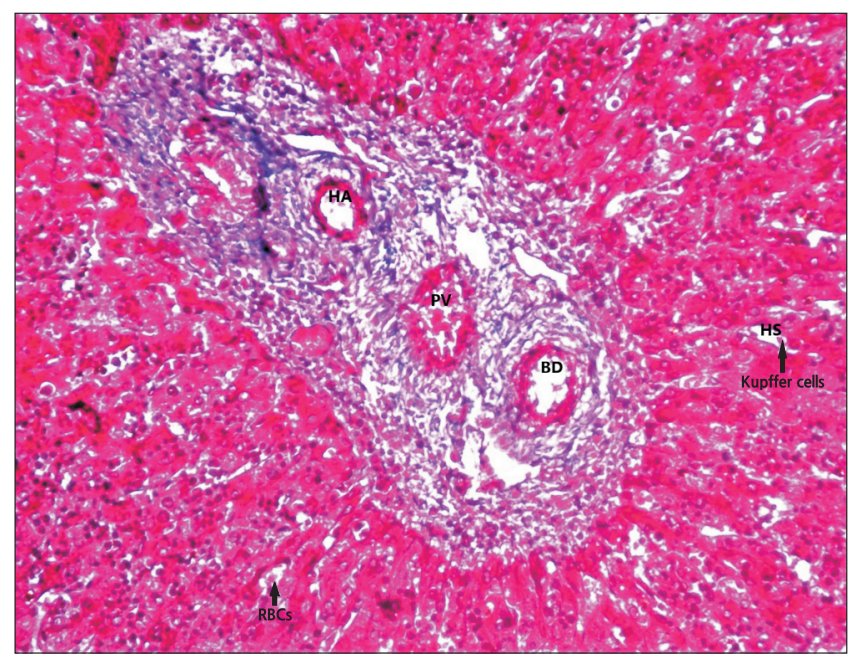

Fig. 3. Photomicrograph (Masson trichrome, $\times 20$ ) of cytology showing Kupffer cells and red blood cells (RBCs) in hepatic sinusoids (HS) along with plates of hepatocytes in 3rd-trimester foetal liver. $\mathrm{HA}$, hepatic artery; PV, portal vein; BD, bile duct.

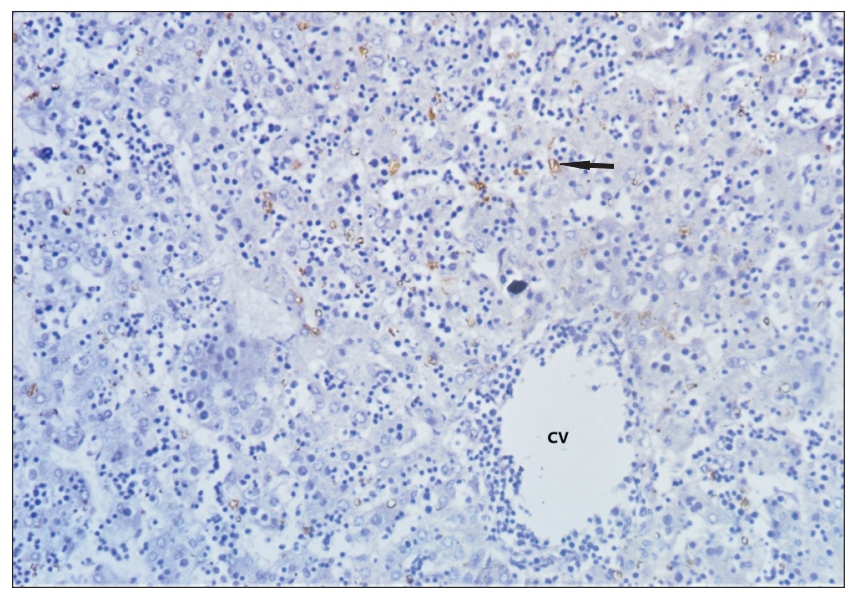

Fig. 4. Photomicrograph $(\times 10)$ of immunohistochemistry with CD30 (Ber-H2): brown discolouration of hepatocytes (arrow) labelled with Ber-H2, indicate immunopositivity. These dark brown staining cells at the edge of two portal tracts shows the formation of normal ductal plate. CV, central vein. within the large portal tract shows no evidence of adjacent ductal plate formation. There are patches of the multilaminar ductal plate with a few lumina. Abundant stromal cells highlighted by antigen can be seen. Biliary canaliculi are also evident, particularly in the vicinity of the ductal plate.

\section{Ultrastructural study}

A well-developed biliary canaliculus was seen. The hepatocytes contained abundant mitochondria, endoplasmic reticulum with few fat globules. Lysosomal bodies and inclu-

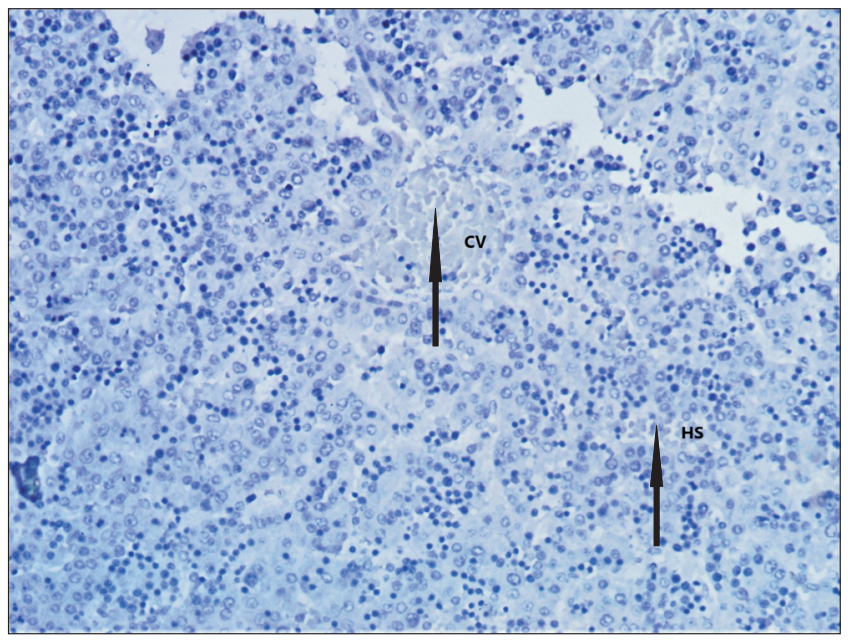

Fig. 5. Photomicrograph $(\times 10)$ of immunohistochemistry with c-myc: brown discolouration of developing central vein (CV), hepatic sinusoids (HS) labelled with c-myc indicates immunopositivity which is more prominent than the Ber-H2.

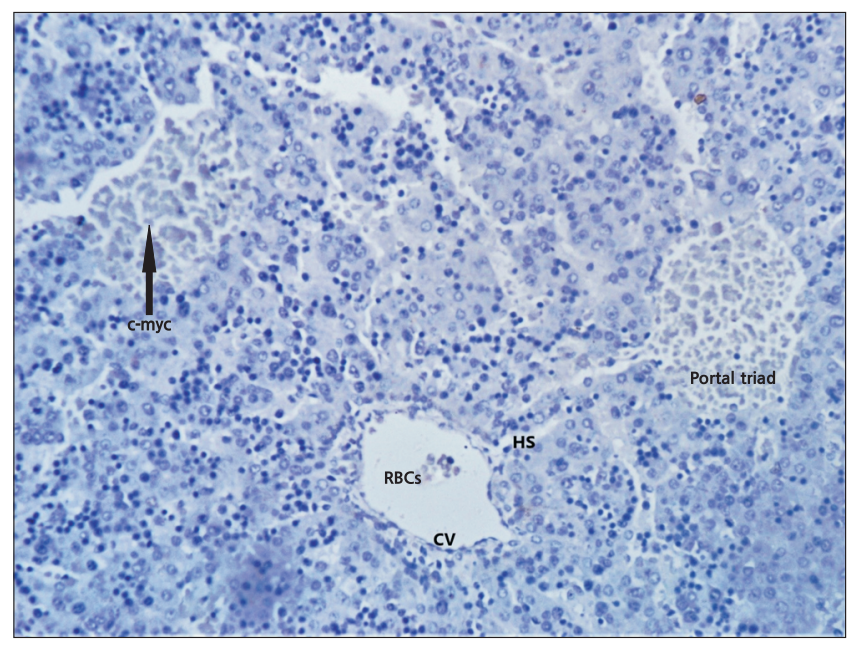

Fig. 6. Photomicrograph $(\times 10)$ of immunohistochemistry with Ki67: brown discolouration of developing hepatic parenchyma indicates immunopositivity which is of similar intensity as c-myc. RBCs, red blood cells; HS, hepatic sinusoids; CV, central vein. 


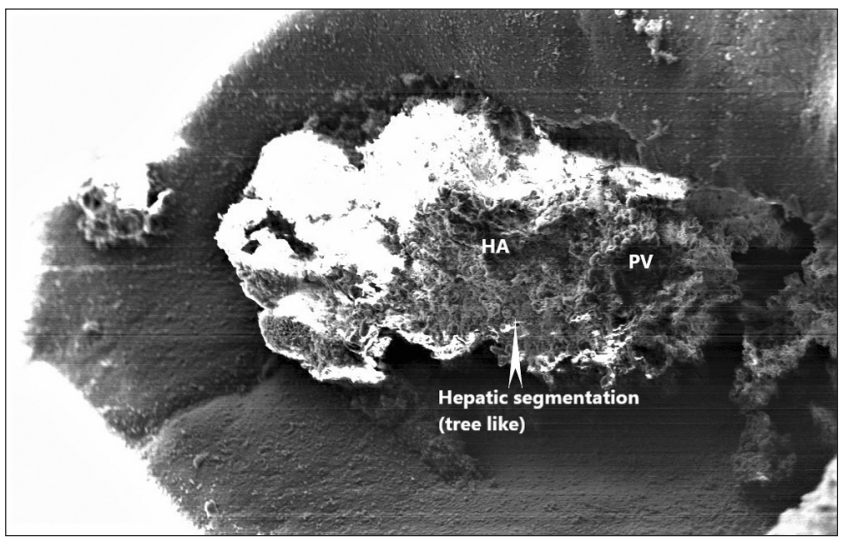

Fig. 7. Photomicrograph of the ultrastructure of the foetal liver obtained through scanning electron microscopy: showing tree-like branching of a portal canal indicating hepatic segmentation of liver. HA, hepatic artery; PV, portal vein.

sion with an amorphous matrix and flecked dense cloudy substance forming tree-like branching of a portal canal indicated hepatic segmentation of liver (Fig. 7). Dense cytoplasmic inclusions appear around the bile canaliculus. At the epithelial-mesenchymal interface, portions of two epithelial cells contained lipid droplets, associated dense particles, multivesicular bodies, microbodies, endoplasmic reticulum, and mitochondria. Portions of two mesenchymal cells were in intimate contact with the epithelial cells at several places, with adherent junctions between the two cell types at several places. Two intercellular junctions (desmosomes) between an epithelial cell and mesenchymal was also seen.

Extracellular fibrous connective tissue elements were found in the intercellular space between an epithelial and mesenchymal cell. Sinusoids containing yolk sac-derived polychromatophilic erythroblasts was seen. Hepatic epithelial cells in contact with cells of the mesenchyme and presumptive hemocytoblasts were seen. Epithelial cells and mesenchyme at the expanding margin of a hepatic parenchymal cord were seen. A free-lying, rounded hemocytoblast lied at the interface. Two presumptive proerythroblasts were incorporated within the epithelial tissue. A hepatic cord and adjacent sinusoid in the central zone were seen. Hepatic epithelial cells and their cytoplasmic processes separating the proerythroblasts could be visualized. Large nucleoli dominate the proerythroblast nuclei. Both yolk sac polychromatophilic erythroblasts and hepatic proerythroblasts lying within the endothelium lined sinusoid were seen. Proerythroblast apparently in a passage between hepatic cord and sinusoid was seen through a gap in the tenuous endothelial layer.
A megakaryocytic precursor was identified by its characteristic granules and cisternal Golgi system, apparently in transit from cord to sinusoid. A thin rim of heterochromatin enveloped the nucleolus. Strands of similar material laced through the nucleolus, while larger blocks of condensed chromatin were distributed in the nucleoplasm. Peri chromatin granules lie both in the nucleoplasm and in the nucleolus. Densely packed ribosomes along with rare and attenuated elements of the endoplasmic reticulum occupied the cytoplasm. Extensive heterochromatinization and shrinkage of the nucleolus accompanied an overall reduction in cellular size. Numerous polyribosomes were disposed of in a finely granular cytoplasmic matrix. A small, residual Golgi system was also seen.

\section{Discussion}

The present work has been undertaken keeping in view the development of the liver and its histological changes during the foetal period. The liver is involved in metabolic, exocrine and endocrine functions. It is unique in its status that it acts as a hematopoietic organ.

The hepatic lobule is the structural unit of the liver, most of the authors described this as a hexagonal structure with a vein placed in the centre. The boundaries of such lobule are clearly defined by connective tissue only in few species like pigs and are sparse in humans with a cord-like arrangement of hepatocytes that branch and anastomose enclosing sinusoids in between. Unlike the adult liver, the hepatic lobular, portal lobular and acinus pattern were not much recognizable except for an ill-defined pattern of hepatic lobule noticed in some areas [8].

The development of the liver occurs gradually, the lobular formation in the liver starts between 9 and 12 weeks of gestation in different stages $[6,7]$. Whereas, portal triad, central vein with cord-like arrangements of hepatocytes are recognizable at around 24 weeks of gestation. Zorn (2008) [9] in his study has observed that the central vein starts appearing at 16th to 17th week of gestation. Functionally, the foetal liver is unique as it acts as a hematopoietic organ. Sinusoidal pattern, hematopoietic cells and Kupffer cells are recognizable in the early stage of fetogenesis. As the gestational age advances hemopoietic cells decreases in number and being replaced by Kupfer cells [10]. The presence of Kupffer cells in the later stages of fetogenesis has also been described by Naito et al. (1997) [11], who found that these macrophages 
develop in the yolk sac and later migrates to the foetal liver. According to Lipp (1952) [12], liver haemopoiesis regresses by 28 to 32 weeks of gestation, once bone marrow haemopoietic function starts. The intrahepatic arterial radicles and branches of the portal vein appear within the liver parenchyma at 10 weeks of gestation [13]. Initially, these arterial radicles are located in the centre of the foetal liver, later they reach the periphery of the liver at 15 weeks [14]. The intrahepatic capillaries which are developed from embryonic vessels are lined by continuous endothelium at 8 weeks of gestation, later they are differentiated into sinusoids with fenestrated endothelium at 17 weeks.

In the present study, cytology and immunohistochemistry showed the development of duct patterns and hemopoietic patterns in all the stages of fetogenesis. The normal ductal plate was marked by the layer of dark brown staining cells at the edge of two portal tracts, multilaminar in places and contains a few lumina. The connective tissue and other structures of the portal tract were not specifically stained. A central vein was seen at the top left and bottom right. Hepatocytes with medium density brown staining were evident between the two portal tracts and the central veins. Erythrocytes were seen in the hepatic sinusoids. Discontinuous, mostly unilaminar ductal plates were found around four portal areas of varying sizes. The bile ductule within the large portal tract showed no evidence of adjacent ductal plate formation. There were patches of the multilaminar ductal plate with a few lumina. Abundant stromal cells highlighted by antigen could be seen. Biliary canaliculi were also evident, particularly in the vicinity of the ductal plate. The presence of haemopoietic cells along with sinusoids and aggregation of hepatocytes were observed in a specimen of 5 to 6 weeks of gestational age, suggestive of commencement of haemopoiesis at about the 6th week of intrauterine life. Haemopoiesis was seen prominently in all the stages studied but a gradual decrease in haemopoiesis was found from 24th to 30th weeks of gestation, and after 32nd weeks, scanty foci of haemopoietic tissues were seen. According to Zamboni (1965) [15] haemopoiesis in the liver becomes fully established around 3rd month of intrauterine life. Whereas, Hamilton et al. (1978) [16] stated that haemopoiesis begins very early in developing liver and reaches its peak at 6 th to 7 th month of foetal life and then regresses up to full term $[17,18]$.

The findings in the present study are in agreement with previous literature [19]. Specimen with 20 weeks of gestational age showed early stage of reticular fibres along with
Kupffer cells. The development of Kupffer cells and connective tissue cells began at about 3rd month of gestational age, thus a delay was observed in the appearance of Kupffer cells. At 24 weeks of gestational age, a portal triad with central vein and sinusoids surrounded by periportal connective tissue was observed. Previous researchers $[11,20]$ reported periportal connective tissue surrounding the bile duct system at around 8 to 12 weeks of gestational age, so, delayed formation of the bile duct system was seen in the present study. The delay in the formation of sinusoids and Kupffer cells along with the biliary duct systems may results in histopathological and developmental abnormalities in the adult liver. The hemopoietic cells were seen between 15 to 35 weeks of gestation. In the present study, portal triad, binucleated hepatocytes, Kupffer cells, portal canal, central vein and sinusoids were observed at 36 weeks of gestation. The haematopoietic function ceased abruptly at 35 weeks of gestation [21].

Previously researchers have studied the cytoarchitecture of foetal liver of various gestational ages. Early work done by Lipp (1952) [12] stated that hepatic parenchymal plates develop from the cell cord organized along their nutrient vessel. The authors also described the uniform pattern of hepatic parenchymal cells throughout the liver and also demonstrated an anastomosing cord-like pattern at places as described by Severn (1972) [13]. Balis et al. (1964) [21] suggested that the liver plates are formed before the development of sinusoids. Potter and Craig (1975) [22] reported that the liver differentiates into masses and plates of cells at the 4th week.

Notenboom et al. (1996) [23] found that lobular pattern is not well defined with cells arranged in anastomosing cord pattern with single-cell thickness. They also stated that the foetal period is crucial for normal liver development with differentiation into adult phenotype from embryonic foregut being a multistep process. Payushina (2012) [24] found fibroblast and myofibroblast around the portal triad and confirmed their finding immunohistochemically. Fibrous tissue along the central vein and portal triad was noticed. The hepatic artery and portal vein were found to be surrounded by fibrous connective tissue. Villeneuve et al. (2009) [14] described that the foetal hepatocytes were arranged in anastomosing sheets separated by capillaries. Emura et al. (1983) [19] noticed the different erythrocytes series in the hemopoietic system during the early stage of hepatic hemopoiesis. The presence of Kupffer cells has also been described by Naito et al. (1997) [11] who found that macrophage develops in the 
yolk sac and migrates to the foetal liver.

In the present study, electron microscopy (EM) showed tree-like branching of a portal canal depicting hepatic segmentation of the foetal liver. Hepatic plates were seen to run from a terminal portal canal toward a terminal hepatic venule. Portal vein and hepatic artery were clearly identified in the scanning EM. The ultrastructure of the foetal liver also showed well-developed biliary canaliculus with hepatocytes containing abundant mitochondria.

Balis et al. (1964) [21] reported periportal bile tubules as the earliest organized structures formed by the association of both hepatocytes and cholangiolar cells arranged around venous channels, suggestive of hepatic segmentation. According to them, the wall of the bile canaliculi is composed of cells with apparently high metabolic activity (well-developed Golgi complex, relatively numerous peribiliary bodies). Any delay in hepatic segmentation will affect the cellular organisation of hepatic parenchyma and will eventually cause a defect in the functions of the liver.

EM also showed glycogen deposits to appear later around 29 to 32 weeks of gestation as fine white specks with vacuolation in the hepatocyte cytoplasm around the developing central vein. While Valdes-Dapena (1957) [25] showed glycogen vacuolization of epithelial cells which is a feature of the foetal liver of the last weeks of gestation. To date, there is no foetal liver transplantation in patients with cirrhosis and hepatocellular carcinoma due to the inadequate knowledge of the descriptive histological architecture of the human foetal liver. The sole treatment available is using human umbilical cord mesenchymal stem cell-derived extracellular vesicles, but these have potential tumorigenic effects or trigger cancer in a patient through upregulating the $\mathrm{Bcl}-2$ oncogene or activating ERK1/2 phosphorylation [26]. The findings of the presents study will help the liver transplant surgeons to understand the cytoarchitecture of foetal liver and future researchers working on hepatic tissue engineering $[27,28]$.

Further studies on a large sample are required to give significant reference data set about the cytoarchitecture of the liver during development. The Mass Spectrophotometry to compare the cytoarchitecture of the normal foetal liver with pathological one (cirrhosis or hepatocellular carcinoma) and micrometry by Image J Software has to be done to show the number of immunopositivity cells in the field.

In conclusion, the organizational changes in lobular pattern, duct pattern and microstructure of liver during fetogenesis are very crucial to achieve the adult morphology in feature. Histogenesis of the foetal liver follows a multistep process depending upon the gestational age, any deviation from normalcy may lead to structural and functional abnormality later in life. So, a clear understanding of the biliary pattern during the fetogenesis as obtained through histochemical study and ultrastructure of hepatic parenchyma will be of immense help in diagnosis and therapeutic segmental resection.

\section{ORCID}

Arpan Haldar: https://orcid.org/0000-0003-4830-038X

Manisha Rajanand Gaikwad:

https://orcid.org/0000-0002-6512-3787

Apurba Patra: https://orcid.org/0000-0002-1107-4481

Soumya C. Bhattacharya:

https://orcid.org/0000-0001-8550-8791

\section{Author Contributions}

Conceptualization: AH, MRG. Data acquisition: AH, MRG. Data analysis or interpretation: AH, MRG, AP. Drafting of the manuscript: AH, MRG, AP, SCB. Critical revision of the manuscript: AH, MRG, AP, SCB. Approval of the final version of the manuscript: all authors.

\section{Conflicts of Interest}

No potential conflict of interest relevant to this article was reported.

\section{Acknowledgements}

Authors thank EM Facility, SAIF, Department of Anatomy, All India Institute of Medical Sciences, New Delhi and IHC Lab, Department of Anatomy (IHC) and Department of Obstetrics and Gynaecology, All India Institute of Medical Sciences, Bhubaneswar.

\section{References}

1. Michalopoulos GK. Liver regeneration. J Cell Physiol 2007;213:286300.

2. Sadler TW. Langman's medical embryology. 7th ed. Philadelphia: Lippincott Williams \& Wilkins; 2005. p. 198-201.

3. Gil MN, Choi DR, Yu KS, Jeong JH, Bak DH, Kim DK, Lee NS, Lee JH, Jeong YG, Na CS, Na DS, Ryu KH, Han SY. Rhus 
verniciflua Stokes attenuates cholestatic liver cirrhosis-induced interstitial fibrosis via Smad3 down-regulation and Smad7 upregulation. Anat Cell Biol 2016;49:189-98.

4. Ajmani ML. Practical embalming. In: Ajmani ML, editor. Text Book Principles of Embalming Techniques in Autopsied Body. New Delhi: Jaypee Brothers Publishers; 1993. p.131-4.

5. Naik KS, Lokanadham S, Gurushanthaiah M. Different gestational age related histogenesis of human foetal liver. Int J Anat Res 2020;8:7408-11.

6. Terada T, Nakanuma Y, Ohta G. Glandular elements around the intrahepatic bile ducts in man; their morphology and distribution in normal livers. Liver 1987;7:1-8.

7. Kieusseian A, Brunet de la Grange P, Burlen-Defranoux O, Godin I, Cumano A. Immature hematopoietic stem cells undergo maturation in the fetal liver. Development 2012;139:3521-30.

8. Jaiswal A, Sinha DN, Singh AK. A study on histology of fetal liver. Natl J Clin Anat 2015;4:26-9.

9. Zorn AM. Liver development. In: Harvard Stem Cell Institute, editor. StemBook. Cambridge: Harvard Stem Cell Institute; 2008.

10. Moore KL, Persaud TVN, Torchia MG. The developing human: clinically oriented embryology. 8th ed. Philadelphia: Elsevier Saunders; 2008. p. 211-42.

11. Naito M, Hasegawa G, Takahashi K. Development, differentiation, and maturation of Kupffer cells. Microsc Res Tech 1997;39:350-64.

12. Lipp W. [The early structural development of the liver parenchyma in man]. Z Mikrosk Anat Forsch 1952;59:161-86. German.

13. Severn CB. A morphological study of the development of the human liver. II. Establishment of liver parenchyma, extrahepatic ducts and associated venous channels. Am J Anat 1972;133:85-107.

14. Villeneuve J, Pelluard-Nehme F, Combe C, Carles D, Chaponnier C, Ripoche J, Balabaud C, Bioulac-Sage P, Lepreux S. Immunohistochemical study of the phenotypic change of the mesenchymal cells during portal tract maturation in normal and fibrous (ductal plate malformation) fetal liver. Comp Hepatol 2009;8:5.

15. Zamboni L. Electron microscopic studies of blood embryogenesis in humans. II. The hemopoietic activity in the fetal liver. J Ultrastruct Res 1965;12:525-41.

16. Hamilton WJ, Boyd JD, Mossman HW. Human embryology: prenatal development of form and function. 4th ed. London: Macmillan; 1978. p. 339-48.
17. Slayton WB, Juul SE, Calhoun DA, Li Y, Braylan RC, Christensen RD. Hematopoiesis in the liver and marrow of human fetuses at 5 to 16 weeks postconception: quantitative assessment of macrophage and neutrophil populations. Pediatr Res 1998;43:774-82.

18. Couvelard A, Scoazec JY, Dauge MC, Bringuier AF, Potet F, Feldmann G. Structural and functional differentiation of sinusoidal endothelial cells during liver organogenesis in humans. Blood 1996;87:4568-80.

19. Emura I, Sekiya M, Ohnishi Y. Two types of immature erythrocytic series in the human fetal liver. Arch Histol Jpn 1983;46:631-43.

20. Miranda RN, Omurtag K, Castellani WJ, De las Casas LE, Quintanilla NM, Kaabipour E. Myelopoiesis in the liver of stillborns with evidence of intrauterine infection. Arch Pathol Lab Med 2006;130:1786-91.

21. Balis JU, Chan A, Conen PE. Electron microscopy study of the developing human liver. Tijdschr Gastroenterol 1964;7:133-47.

22. Potter EL, Craig JM. Weights standards for organs for early human fetuses. In: Potter EL, Craig JM, editors. Pathology of the Fetus and the Infant. 3rd ed. Chicago: Year Book Medical Publishers; 1975. p.15-24.

23. Notenboom RG, de Boer PA, Moorman AF, Lamers WH. The establishment of the hepatic architecture is a prerequisite for the development of a lobular pattern of gene expression. Development 1996;122:321-32.

24. Payushina OV. Hematopoietic microenvironment in the fetal liver: roles of different cell populations. Int Sch Res Not 2012;2012:979480.

25. Valdes-Dapena MA. An atlas of foetal and neonatal histology. Philadelphia, PA: J.P. Lippincott Co; 1957. p. 75-87.

26. Abbaszadeh H, Ghorbani F, Derakhshani M, Movassaghpour A, Yousefi M. Human umbilical cord mesenchymal stem cellderived extracellular vesicles: a novel therapeutic paradigm. J Cell Physiol 2020;235:706-17.

27. Giancotti A, Monti M, Nevi L, Safarikia S, D’Ambrosio V, Brunelli R, Pajno C, Corno S, Di Donato V, Musella A, Chiappetta MF, Bosco D, Panici PB, Alvaro D, Cardinale V. Functions and the emerging role of the foetal liver into regenerative medicine. Cells 2019;8:914.

28. El Baz H, Demerdash Z, Kamel M, Hammam O, Abdelhady DS, Mahmoud S, Hassan S, Mahmoud F, Atta S, Riad NM, Gaafar T. Induction of hepatic regeneration in an experimental model using hepatocyte-differentiated mesenchymal stem cells. Cell Reprogram 2020;22:134-46. 